\title{
Special issue: Management of road and railway traffic and transportation engineering
}

(C) The Author(s) 2017. Published by Higher Education Press. This is an open access article under the CC BY license (http:// creativecommons.org/licenses/by/4.0)

In recent years, with the development of road and railway transportation industries, a variety of complicated decisionmaking problems have emerged in real-world applications. It is urgent to analyze these problems from the perspective of theoretical and methodological innovations, and provide methods in management, decision-making and application so as to achieve efficient operations of traffic and transportation systems. These problems have also attracted considerable attention from both theoretical researchers and practical engineers. All the relevant studies intend to develop effective and efficient approaches for well operations of the road and railway traffic systems.

This issue aims to cover all aspects, including management, optimization, control, and other related techniques, to determine effective solutions/policies and assist in making the most possible decision making in the field of road and railway traffic and transportation engineering. It also provides a forum for the latest and significant research in designing, planning, and managing of complex transportation systems. Moreover, this issue intends to provide a platform for utilization of theoretical results to support reasonable decision-making in related fields of traffic and transportation. Relevant topics focus on theories and applications of traffic and transportation management in road and railway transportation industries.

After we announced the Call-for-Paper in December 2016, this special issue has attracted much attention from many researchers. After a rigorous review, only the following research papers were finally accepted for publication. Detailed information is given below.

Chen et al. propose five indices based on the emerging on-demand ride service data to evaluate the urban network-wide travel time reliability. These indices consider the probability distribution of the travel time rate of each origin-destination pair in the road network. For demonstration, they analyze the network-wide travel time reliability in Beijing from time and space dimensions. Their proposed indices provide effective tools for the comprehensive evaluation of the road network traffic dynamics and day-to-day travel time variations.

$\mathrm{Li}$ and Li present an improved multiclass dynamic network traffic flow propagation model, which takes into account physical queues. In their model, each involved link is particularly decomposed into free flow area and queue area, where same-class vehicles are required to satisfy the first-in-first-out principle on the whole link, and the differentclass vehicles follow the first-in-first-out principle in the queue area but not in the free flow area. Numerical experiments are implemented to demonstrate the performance of their model.

Wang et al. utilize a stochastic constrained shortest path approach to investigate the robust train speed trajectory optimization. They propose a distance-speed network on an interstation and use discrete sample-based random variables with correlation to represent the uncertainty of energy consumption. A stochastic constrained shortest path model with travel time threshold constraints is formulated to minimize the expected total energy consumption. To solve the model, they propose a dynamic programming based on Lagrangian relaxation algorithm, whose effectiveness is demonstrated by a series of numerical experiments.

Yin et al. investigate the metro train rescheduling problem under disrupted scenarios through adding backup trains. They first formulate a mixed integer programming model to specify the optimal train rescheduling strategies by

Received October 11, 2017 
considering both in-service and backup trains. Given the nonlinearity of their proposed model, they further adopt linearization methods to obtain the equivalent linear model. Experiments are carried out to demonstrate the effectiveness of their approaches.

Lin and Kumar propose a novel concept called IN2CLOUD for collaborative management of large railway data. The concept is developed with assumption that organizations want to learn from one another, but they do not want to share their raw data or information. This concept aims to achieve the global optimization of railway industry systems in a collaborative way. Their developed solutions will enhance business security, economic sustainability, and decision support in a large railway data management system.

$\mathrm{Ng}$ et al. investigate the robustness of various public-private partnership (PPP) configurations based on their previous works. They analyze the effects of PPP configurations on stakeholders' risks and returns with the consideration of population or demand growth and railway construction cost uncertainties. They also examine the eventual outcome of particular PPP configurations and answer some questions with respect to this topic. Their approach is significant for managing risks and facilitating the formation of appropriate PPP for railway and housing development.

Yu et al. optimize the urban bus operation frequency under the common route condition with rail transit. They propose the overlapped operation conditions of bus and rail transit through a two-level model from the perspective of bus operators, in which the upper-level model is formulated with respect to frequency optimization and solved by the shuffled complex evolution method. The lower-level one is associated with logit model based on agent simulation. The experiment with practical data is used to show the advantages and feasibility of the proposed approaches.

Lin et al. explicitly discuss the governance potential of major countries in the high-speed rail value chain and investigate the possible methods and strategies to improve the high-speed railway governance in China. A series of insightful findings are proposed in their study.

Xie et al. calibrate a mathematical model with the collected pedestrian flow and speed data to characterize the relationships between relevant macroscopic quantities to provide insight regarding pedestrian behaviors at the carnival event. The results of the model can further understand the crowd behavior under different conditions at carnival events and help improve the safety and efficiency of similar events in the future.

Auer et al. investigate the process from receiving raw probe data to space-time diagrams and the resulting traffic service. In this process, all raw position data received from global positioning system (GPS) should be first matched in a digital road map. After the matching process, space-time diagrams are created to illustrate traffic situation details over space and time. The examples illustrate how traffic service quality depends on the number of matched GPS raw data.

Finally, we would like to thank all the authors for contributing their valuable studies to the special issue of Frontiers of Engineering Management. We also sincerely thank the Editor-in-Chief and all the reviewers for their kind supports in the process of organization and review for this special issue.

\section{Guest Editors-in-Chief}

Yongfu SUN

Academician, Chinese Academy of Engineering, China

Ziyou GAO

Professor, Beijing Jiaotong University, China

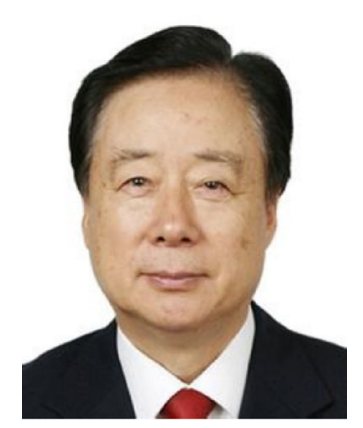

Yongfu SUN, an academician in the Chinese Academy of Engineering, is an expert in railway engineering and the Chairman of China Railway Society. He has been studying railway construction techniques and managements for several decades. To formulate a new system that adapts to market economy, he studied the reformation mechanism of railway construction management after he served as a Vice Minister of the Ministry of Railways in China in 1984. He investigated the decision-making system of railway construction projects and proposed new planning and design ideas of constructing large railway corridors according to system engineering.

He led and finished many important engineering projects in China, such as Daqin, Jingjiu, Nankun, Baozhong, Hengguang, and Lanxin. He summarized a series of new technologies on mountain railway construction and successfully developed heavy-haul transportation equipment for Daqin railway. He explored the high-speed railway technology and formulated relevant standards and specifications. After 2001, he led the construction of the Qingzang railway and made significant contributions in building this railway into a world-class plateau railway through management innovation and technological innovation. 


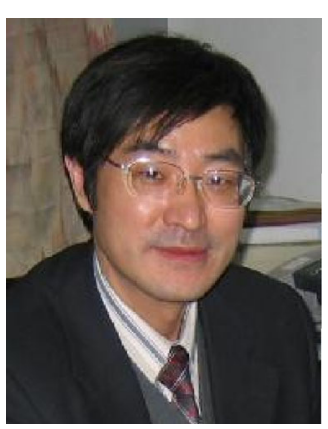

Ziyou GAO is a professor in the School of Traffic and Transportation, Beijing Jiaotong University. He received his Ph.D. degree in operations research and control theory from the Chinese Academy of Sciences in 1994. From October 1993 to September 1995, he was a postdoctoral researcher majoring in transportation management in Beijing Jiaotong University, where he was previously a professor and later a doctor supervisor. He is currently the dean of the Institute of Transportation System Science and Engineering.

Dr. GAO is also the Chairman of the Society of Management Science and Engineering of China, Vice Chairman of Chinese Society of Optimization, Overall Planning and Economical Mathematics, Vice Chairman of Systems Engineering Society of China, a coeditor of Traffic and Transportation Systems Engineer and Information, a member of the Editorial Board of Transportmetrica, and a member of the Editorial Advisory Board of Transportation Research Part $B$. He was also elected as a foreign member of the Russian Academy of Natural Sciences in 2003. His research interests include transportation planning and management. He has published more than 400 papers in conferences, and major journals of his field. 